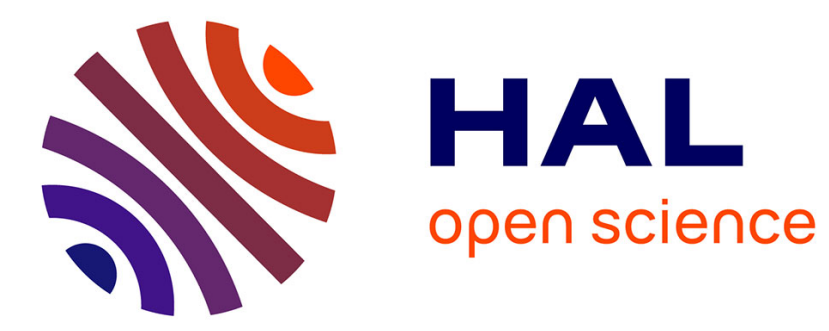

\title{
La datation dans les inscriptions médiévales françaises
}

Robert Favreau

\section{To cite this version:}

Robert Favreau. La datation dans les inscriptions médiévales françaises. Bibliotheque- Ecole des Chartes, 1999, 157 (1), pp.11-39. 10.3406/bec.1999.450960 . halshs-03206293

\section{HAL Id: halshs-03206293 \\ https://shs.hal.science/halshs-03206293}

Submitted on 23 Apr 2021

HAL is a multi-disciplinary open access archive for the deposit and dissemination of scientific research documents, whether they are published or not. The documents may come from teaching and research institutions in France or abroad, or from public or private research centers.
L'archive ouverte pluridisciplinaire HAL, est destinée au dépôt et à la diffusion de documents scientifiques de niveau recherche, publiés ou non, émanant des établissements d'enseignement et de recherche français ou étrangers, des laboratoires publics ou privés. 


\section{La datation dans les inscriptions médiévales françaises}

Robert Favreau

\section{Citer ce document / Cite this document :}

Favreau Robert. La datation dans les inscriptions médiévales françaises. In: Bibliothèque de l'école des chartes. 1999, tome 157, livraison 1. pp. 11-39;

doi : https://doi.org/10.3406/bec.1999.450960

https://www.persee.fr/doc/bec_0373-6237_1999_num_157_1_450960

Fichier pdf généré le 08/11/2019 


\title{
Résumé
}

Un premier essai de synthèse sur l'expression de la date dans les inscriptions médiévales françaises amène à envisager successivement le comput des années (années consulaires, années de règnes et, surtout, ère de l'Incarnation), les périodes chronologiques (principalement l'indiction), l'emploi du calendrier romain et son remplacement par le quantième du mois et, enfin, la datation par une fête religieuse. L'enquête dégage de fréquentes différences entre France du nord et France du sud, et un jeu de concordances et discordances avec les usages diplomatiques, historiographiques, liturgiques, qui souligne aussi bien la spécificité des sources épigraphiques que la communauté culturelle entre les auteurs de ces créations diverses.

\section{Zusammenfassung}

Eine erste Gesamtschau über die verschiedenen Möglichkeiten der Datumsdarstellung in der mittelalterlichen französischen Epigraphik behandelt folgende Themen : Die Jahreszählung (das Konsulatsjahr, das Herrschaftsjahr und den - vorherrschenden - Inkarnationsstil) ; die chronologischen Perioden (allen voran die Indiktion); der Gebrauch des römischen Kalenders und sein Ersatz durch die numerische Zählung der Monatstage ; schließlich die Datierung durch Kirchenfeste. Die Untersuchung fördert wiederholt Unterschiede zwischen Nord- und Südfrankreich zutage. Weiterhin offenbart sie ein Geflecht von Ubereinstimmungen und Verschiedenheiten der vorgefundenen Datumsdarstellungen mit diplomatischen, historiographischen und liturgischen Gebräuchen, was sowohl die wechselseitige kulturelle Verbundenheit der Autoren dieser verschiedenen Darstellungstraditionen zeigt als auch die Eigenarten der epigraphischen Quellen hervortreten läßt.

\begin{abstract}
This first attempt to assess the dating methods used in French mediaeval inscriptions considers, in turn, the computation of years (consular, regnal, and, more prominently, years of the era of the Incarnation), the chronological periods (indictions mainly), the use made of the Roman calendar with its eventual superseding by the day of the month, and, lastly, dating by feasts of the Church. The inquiry points to frequent differences in French practice between North and South ; and, with respect to diplomatic, historiographical and liturgical customs, to a set of concordances and discordances that brings out both the specificity of epigraphical sources and the cultural kinship shared by the authors of the various kinds of documents.
\end{abstract}




\title{
LA DATATION DANS LES INSCRIPTIONS MÉDIÉVALES FRANÇAISES
}

\author{
par \\ ROBERT FAVREAU
}

Marquées par une tradition et des fins spécifiques, mais entretenant un dialogue incessant avec les productions diplomatiques, historiographiques, liturgiques, les sources épigraphiques fournissent un matériau considérable sur les systèmes médiévaux d'expression du temps. Une véritable synthèse, en ce domaine encore très largement en friche, ne pourra être réalisée qu'après l'achèvement du Corpus des inscriptions de la France médiévale. Le présent essai n'est qu'une introduction, établie à l'occasion d'un travail parallèle des épigraphistes des universités de Poitiers et de Munich, et présentée lors du congrès d'épigraphie médiévale qui s'est tenu à Rome en septembre 1993 '. Il s'appuie sur les seize volumes publiés à cette date par le Corpus des inscriptions de la France médiévale, sur des missions photographiques déjà réalisées sur le terrain pour un certain nombre d'autres départements, et, pour les autres départements comme pour les inscriptions des $\mathrm{XIV}^{\mathrm{e}}$ et $\mathrm{XV}^{\mathrm{e}}$ siècles, qui ne sont pas encore prises en compte par le Corpus, sur le fichier général tenu à Poitiers et régulièrement enrichi. Sa seule ambition sera de fournir quelques repères à l'épigraphiste comme à l'historien familier d'autres sources, et de provoquer une attention renouvelée à l'évolution générale et aux particularités locales de la datation dans les inscriptions.

1. La notation des années. - Comme l'on pouvait s'y attendre, les auteurs d'inscriptions font appel à un large spectre d'ères et d'expressions, des plus savantes aux plus communes, quand il leur faut qualifier une année. Les chronologistes juifs et chrétiens s'étaient efforcés d'établir, à partir des indications de la Bible, l'année de la création du monde; ces calculs sont con-

1. Les actes du congrès, dont la publication avait été envisagée par l'Université de Rome, n'ont jamais paru. L'équipe de Munich a donné les résultats de son enquête en 1996 : Maria Glaser et Franz-Albrecht Bornschlegel, Datierungen in mittelalterlichen Inschriften des deutschen Sprachraumes, dans Archiv für Diplomatik, Schriftgeschichte, Siegel-und Wappenkunde, t. 42, 1996, p. 525-556.

Robert Favreau, professeur honoraire à l'université de Poitiers, 6 rue Montgautier, F-86000 Poitiers. 
nus notamment des auteurs qui rédigent des chroniques universelles, avec des variantes selon qu'ils ont suivi les ères mondaines d'Alexandrie, d'Antioche ou de Constantinople ${ }^{2}$. Cet usage n'a jamais été introduit dans les actes en Occident ${ }^{3}$, et ne se rencontre pas dans les inscriptions médiévales françaises. Deux inscriptions au moins en Italie l'ont utilisé, l'une à Rome, à Sant'Angelo in Pescheria au VIII siècle, "anno ab initio mundi sex millia ducentos sexaginta tres... " ${ }^{4}$, l'autre à San Martino d'Agricena, en Italie $\mathrm{du}$ sud, au XII ${ }^{\mathrm{e}}$ siècle, « in anno sex milleno mundi machine minus uno.... ${ }^{5}$.

Du IV e au VII ${ }^{e}$ siècle, les inscriptions chrétiennes de la Gaule emploient la datation à partir des consuls romains. Ceux-ci n'eurent guère plus, dès le début $\mathrm{du} \mathrm{IV}^{\mathrm{e}}$ siècle, que des prérogatives honorifiques, dont celle de donner leur nom à l'année qui avait vu leur élection. On gravait les noms des consuls de l'année sur les diptyques d'ivoire que l'on envoyait dans les provinces, et la loi romaine, notamment la novelle 47 de Justinien, prescrivait de dater les actes publics de l'année consulaire. Le dernier des consuls fut, en Orient, en 541, Flavius Basilius Junior. On data ensuite pendant vingtcinq ans les actes des années " après le consulat de Basilius », jusqu'à ce que Justin le Jeune eût pris, le $1^{\text {er }}$ janvier 566 , le titre de consul. Dès lors il n'y eut plus qu'un consul perpétuel, l'empereur. Sur les marbres de la Gaule, cependant, on resta fidèle à une datation par les années postérieures au consulat de Basilius : le système s'observe encore en $605^{6}$, alors que l'indication du postconsulat de Justin se maintient jusqu'au VII ${ }^{\mathrm{e}}$ siècle. Les différences régionales peuvent, toutefois, être fortement marquées : à Trèves, deux inscriptions seulement, toutes deux grecques, portent une année de consulat, en 383 et $4.09^{7}$; dans la Viennoise du nord, quatre-vingt-deux inscriptions sont datées d'un consulat ou d'un postconsulat. Le nombre excep-

2. L'art de vérifier les dates des faits historiques, des chartes, des chroniques et autres anciens monuments..., $3^{\mathbf{e}}$ éd. par un religieux bénédictin de la congrégation de Saint-Maur, t. I, Paris, 1773, p. XV-XVII; Comte de Mas Latrie, Trésor de chronologie, d'histoire et de géographie pour l'étude et l'emploi des documents du Moyen Âge, Paris, 1889, col. 30-34.

3. Arthur Giry, Manuel de diplomatique, Paris, 1893, p. 88.

4. Richard Krautheimer, Corpus basilicarum christianarum Romae : le basiliche cristiane antiche di Roma (sec. IV-IX), vol. I, Vatican, 1937, p. 67.

5. Alfredo Petrucci, Cattedrali di Puglia, $2^{\mathrm{e}}$ éd., Rome, 1964, p. $149-150$ et 562 ; Émile Bertaux, $L$ 'art dans l'Ttalie méridionale de la fin de l'Empire romain à la conquête de Charles d'Anjou, rééd., Paris-Rome, 1968 (I ${ }^{\text {re }}$ éd. 1904), t. II, p. 701.

6. Edmond Le Blant, L'épigraphie chrétienne en Gaule et dans l'Afrique romaine, Paris, 1890, p. 12. Le Blant indique le nombre de deux cent trente-neuf inscriptions chrétiennes [de Gaule] datées de dates consulaires ou royales (p. 14).

7. Nancy Gauthier, Recueil des inscriptions chrétiennes de la Gaule antérieures à la Renaissance carolingienne, I, Première Belgique, Paris, 1975, p. 48, et $\mathrm{n}^{\text {os }} 93$ et 211. 
tionnel d'inscriptions datées ainsi en Viennoise peut être considéré comme une « expression manifeste de la persistance de la culture gallo-romaine de cette contrée, car ni à Lyon ni à Arles on n'observe une telle abondance ${ }^{8}$. Naturellement, cette façon de dater a été particulièrement en honneur à Rome 9 .

Peu à peu, aux années de postconsulat, qui étaient de plus en plus dépourvues de signification, on ajouta les années de l'empire ou celles de règne ou de pontificat, tendance qui ne pouvait que se renforcer lorsque l'empereur eut retenu pour lui le consulat, en 567. Pour la Gaule, ce fut à compter de la fin $\mathrm{du} \mathrm{V}^{\mathrm{e}}$ siècle que les inscriptions portèrent les années de règne de rois wisigoths ou francs ${ }^{10}$. Cette façon de dater devint si habituelle qu'elle entra dans les formulaires, comme le montre une inscription où le graveur a oublié de remplacer par l'année de règne le «tanto " trouvé dans son modèle ${ }^{11}$. C'est le premier mode de datation que l'on rencontre dans les inscriptions de l'époque carolingienne, dans la France du sud et de l'ouest : Nice-Cimiez, Estoublon (Alpes-de-Haute-Provence), Sisteron et Vienne, Béziers et Palaja (Aude), Poitiers ${ }^{12}$, Tours, Angers et Bazouges (Mayenne), la France du nord et de l'est n'étant représentée que par une inscription métrique de Reims, "qum juvenis Karolus regeret diademata regni "13. On observe ensuite une interruption d'un siècle, entre 916 et 1012. L'année de règne est alors à nouveau notée jusqu'au XIII ${ }^{e}$ siècle, plus en complément de datation ou par imitation des actes de chancellerie : dix exemples pour le $\mathrm{XI}^{\mathrm{e}}$ siècle, onze pour le XII ${ }^{\mathrm{e}}$, onze également pour le XIII' ${ }^{\mathrm{e}}$. Après une nouvelle interruption de plus d'un siècle, on peut relever deux derniers exemples isolés au XVe siècle, à Béziers et à Bordeaux. Au total, quaranteneuf inscriptions du VII ${ }^{e}$ au $\mathrm{XV}^{\mathrm{e}}$ siècle présentent une datation par l'année de règne ou par la seule mention du règne, la formule la plus courante étant introduite par le "regnante " qui est utilisé dès 779 . Déjà en 876 , à Bazouges, puis en 916, à Palaja, la mention du règne vient seulement en appendice après la référence à l'année de l'Incarnation.

8. Françoise Descombes, Recueil des inscriptions chrétiennes de la Gaule antérieures à la renaissance carolingienne, XV, Viennoise du Nord, Paris, 1985, p. 49.

9. Jean-Baptiste De Rossi, Inscriptiones christianae urbis Romae septimo saeculo antiquiores, pars $1^{a}$, Epitaphia certam temporis notam exhibentia, Rome, 1857-1861, p. XI-LXX.

10. E. Le Blant, L'épigraphie chrétienne..., p. 12-13.

11. E. Le Blant, Manuel d'épigraphie d'après les marbres de la Gaule..., Paris, 1869, p. 59-74, et Sur les graveurs des inscriptions antiques, dans Revue de l'art chrétien, t. 3, 1859, p. 367-368 (marbre de Crussol, Ardèche).

12. Trois inscriptions, dont l'une conservée au Musée national du Moyen Âge à Paris.

13. Gallia christiana, t. IX, col. 40, inscription due à l'archeveque Hincmar; M.G.H., Poetae latini aevi carolini, t. III (Berlin, 1896), éd. Ludwig Traube, p. 409. 
Dans trois exemples postérieurs, le nom du prince est donné avec son numéro d'ordre : "regente in Galliis rege Ludovico septimo " en 1143 à l'abbaye normande Saint-André-en-Gouffern ${ }^{14}$; "regnante imperatore Frederico pronominato primo " en 1189 à Strasbourg 15; "regnante Ludovico rege $I X$ " en 1230 à Saint-Denis ${ }^{16}$. On a un premier exemple d'épithète accolée au nom du roi à Saint-Georges de Roye (Somme) au XI ${ }^{\mathbf{e}}$ siècle, "temporibus gloriosissimi... Francorum regis » 17 ; mais il faut ensuite attendre le $\mathrm{XIII}^{\mathrm{e}}$ siècle pour trouver d'autres exemples, qui font intervenir Philippe Auguste, “ a Deo dato » (1202, Maguelonne), "illustris » (1214, Arras), et Louis IX, « illustrissimo » (1227, Longpont); on observe évidemment la même disposition dans les deux derniers exemples du XVe siècle, "hauta poissansa " pour Charles VI (1448, musée de Béziers), ou " christianissimo principe » (1479, cathédrale de Bordeaux) pour Louis XI. On pourrait joindre à ces datations par les années de règne quelques autres exemples de datation à partir d'un pontificat (pape, évêque) ou d'un principat. D'un point de vue méthodologique, il faut noter à part les exemples isolés, très précoces ou très tardifs, et insister sur les continuités, constatations qui s'appliquent aussi à l'étude de l'écriture.

Le point de départ ordinaire de la chronologie dans les inscriptions médiévales est sans conteste l'an de l'Incarnation. On sait que ce mode de calcul fut mis au point par un moine de la première moitié du vie siècle, Denis le Petit. Dans une chrétienté qui s'affirmait, ce calcul avait l'intérêt de donner comme point de départ de l'année la référence à l'événement qui fondait une ère nouvelle, l'Incarnation du fils de Dieu. Il ne connut d'abord qu'une propagation restreinte; en Gaule, il apparaît pour la première fois dans des capitulaires de 742 et de $744^{18}$, mais la chancellerie ne l'utilisera qu'au cours du règne du roi Eudes, à la fin du IX ${ }^{\mathbf{e}}$ siècle, puis de façon continue à compter de $967^{19}$, à une époque qui est aussi le point de départ

14. Arcisse de Caumont, Statistique monumentale du Calvados, Caen, 1859 (réimpr. Mayenne, 1967), t. IV, p. 709.

15. Robert Will, Répertoire des inscriptions romanes de l'Alsace, dans Revue d'Alsace, t. 98 , 1959 , p. 49-84, à la p. 79 , et Répertoire... : complément, ibid., t. 112,1986 , p. 49-61, à la p. 58 .

16. Jules Formigé, L'abbaye royale de Saint-Denis, recherches nouvelles, Paris, 1960, p. 121.

17. Paul Marchegay, Translation des reliques de saint Florent de Roye à Saumur [fragment d'une histoire manuscrite de l'abbaye de Saint-Florent de Saumur par dom Jean Huynes, religieux de cette abbayel, dans Bibliothèque de l'École des chartes, t. 3, 1841-1842, p. 475-498, aux p. 476 et $481-482$.

18. A. Giry, Manuel..., p. 89.

19. Recueil des actes d'Eudes, roi de France (888-898), publ. par Robert-Henri Bautier, Paris, 1967 (Chartes et diplômes), p. CXI-CXII : premiers emplois isolés à la chancellerie du roi Eudes (à l'imitation de la chancellerie de Charles le Gros), et dans cinq diplômes de Louis IV. 
de son usage à la chancellerie pontificale comme dans les inscriptions romaines (première occurrence pour un acte en 957, pour une inscription en 963) ${ }^{20}$. Annalistes et chroniqueurs l'avaient employé bien avant. Et de fait, dans les inscriptions, on le rencontre en France à partir du deuxième tiers du IXe siècle, si l'on écarte une inscription de Germigny-des-Prés de janvier 806 qui est un faux réalisé entre 1840 et $1846^{21}$. Le premier exemple en serait l'épitaphe d'Adelberge, à Saint-Martin de Tours, dont la date pose question, puisque le texte porte "anno Domini DCCCXXL "; Paul Deschamps a proposé d'y voir une erreur du lapicide et a daté l'inscription de $840^{22}$. L'épitaphe de Dodon, connue par le bénédictin dom Fonteneau, place la mort de cet abbé de Saint-Savin en 853, «anno Incarnationis Domini $D C C C L I I I$ " ${ }^{23}$. La même formule se trouvait sur les inscriptions funéraires de 844 à 868 provenant du cimetière de l'abbaye de Saint-Riquier ${ }^{24}$, comme dans l'épitaphe sur ardoise de Gishwal, en 876, à Bazouges ${ }^{25}$, tandis qu'à Die, pour l'épitaphe d'Arnulfe en 892, on a utilisé l'expression voisine "anno Incarnationis dominicae » 26 . C'est " anno Domini " que l'on trouve à Saunay en Touraine en 874 et à Arles en $883^{27}$. Les derniers des dix-

20. Iiro Kajanto, Dating in the Latin inscriptions of medieval and Renaissance Rome, dans Arctos, acta philologica Fennica, t. 11, 1977, p. 41-61, à la p. 44.

21. Jean Hubert, Germigny-des-Prés, dans Congrès archéologique de France, Orléans, 1930, Paris, 1931, p. 534-568, à la p. 563 (réimpr. dans Nouveau recueil d'études d'archéologie et d'histoire, Genève, 1985 [Mémoires et documents publiés par la Société de l'École des chartes, 29], p. 245-280, à la p. 276), reprenant ici les conclusions de Jacques Soyer, Les inscriptions gravées sur les chapiteaux de Germigny-des-Prés sont-elles authentiques?, dans Bulletin archéologique du Comité des travaux historiques et scientifiques, 1923, p. 197-216.

22. Paul Deschamps, Étude sur la paléographie des inscriptions lapidaires, de la fin de l'époque mérovingienne aux dernières années $d u$ XII' siècle, dans Bulletin monumental, t. 88, 1929 , p. $5-88$, ̀̀ la fig. 2 . Une autre hypothèse serait une datation de 830 .

23. R. Favreau, Les inscriptions de l'église de Saint-Savin-sur-Gartempe, dans Cahiers de civilisation médiévale, t. 19, 1976, p. 9-37, à la p. 10.

24. J. Hubert, Saint-Riquier et le monachisme bénédictin en Gaule à l'époque carolingienne, dans Il monachesimo nell'alto medioevo e la formazione della civiltà occidentale, 8-14 aprile 1955, Spolète, 1957 (Settimane di studio del Centro italiano di studi sull'alto medioevo, 4), p. 293-303, à la p. 298 et pl. III (réimpr. dans Arts et vie sociale de la fin du monde antique au Moyen Âge, Genève, 1977 [Mémoires et documents publiés par la Société de l'École des chartes, 24], p. 373-394, à la p. 378); abbé Henocque, Histoire de l'abbaye et de la ville de Saint-Riquier, Amiens, 1880 (Mémoires de la Société des Antiquaires de Picardie, documents inédits concernant la province, 9), t. I, p. 258 et pl. h.-t. : une inscription dont on ne garde que la date, DCCCXLIIII, et deux de 866 et 868 avec « anno Incarnationis Domini ».

25. P. Deschamps, Étude sur la paléographie..., fig. 5.

26. Corpus des inscriptions de la France médiévale [désormais: C.I.F.M.], 16, Alpes-deHaute-Provence, Hautes-Alpes, Ardèche, Drôme, éd. R. Favreau, Jean Michaud, Bernadette Mora, Paris, 1992, p. 127.

27. May Vieillard-Troiekouroff, Les sculptures et objets préromans retrouvés dans les fouilles 
huit exemples antérieurs à l'an mil se situent à Tours en 913, à Palaja en 916, à Angers en 925, à Err (Pyrénées-Orientales) en 930, à Rouen en 942, à Tannay (Ardennes) en 977, à Saint-Omer en 984 et à Saint-JeanPoutge (Gers) en 990. Ce mode nouveau de datation touche ainsi treize départements. Il est notamment inconnu de la France centrale, de l'Île-de-France, de la France de l'est - Champagne, Lorraine, Alsace, Bourgogne (fig. 1).

Le $\mathrm{XI}^{\mathrm{e}}$ siècle fournit trente-cinq exemples de cette datation dans vingt-deux départements. La France du Centre reste encore absente, l'île-de-France n'est représentée que par un exemple en 1077 à Lagny (Seine-et-Marne), la Bourgogne par deux exemples, à Saint-Bénigne de Dijon en 1027 et à Cîteaux en 1098. Quand on se place à la fin du XI ${ }^{\mathbf{e}}$ siècle, il n'y a encore que vingt-neuf départements où la datation par une année de l'Incarnation ait été utilisée. Cette façon de dater se généralise alors, puisqu'on la trouve dans vingt-deux départements, dont treize nouveaux, au cours de la première moitié $\mathrm{du} X \mathrm{XI}^{\mathrm{e}}$ siècle, et dans trente et un, dont dix nouveaux, dans la première moitié du XIII' ${ }^{e}$ siècle. Le système est alors pratiqué dans soixante-dix départements. France du Centre et ก̂le-de-France sont parmi les régions les plus lentes à adopter l'ère de l'Incarnation dans les inscriptions : dans la région parisienne, on le trouve en 1144 à Saint-Denis. Ainsi ce mode de datation a été pratiqué, en épigraphie, dans le tiers des départements avant la fin du XI ${ }^{e}$ siècle, dans à peine la moitié au milieu du XII ${ }^{\mathrm{e}}$, dans les trois quarts au milieu du XIII' siècle. On doit aussi tenir compte du fait que ces années de l'Incarnation ne commencent pas le $l^{\text {er }}$ janvier, mais à l'Annonciation (25 mars), à Pâques ou à Noël. Dans la région parisienne, le changement de millésime s'effectue à Pâques : une inscription des Célestins prend soin de préciser la date sous la forme " lundi lendemain de Pasques en commençant en l'an $1382 » 28$.

On emploie d'abord la formule "anno Incarnationis Domini (ou) dominice ", que l'on trouve de 853 à 1269 pour la première expression, de 892 à 1221 pour la seconde, avec quelques emplois isolés plus tardifs, respectivement en 1317-1341 et 1430, et en 1352-1455. On trouve ensuite, et de façon concomitante, la formule " anno ab Incarnatione Domini ", régulièrement employée de 1002 à 1286, avec deux exemples isolés en 1324 et en 1329 et deux autres en 1428 et 1478 , ou, plus rarement, l'expres-

de 1860 et de 1886 à Saint-Martin de Tours, dans Cahiers archéologiques, t. 13, 1962, p. 85-118, à la p. 117, note 5; R. Ranjard, La Touraine archéologique, Mayenne, 1975, p. 641 ; C.I.F.M., 14, Alpes-Maritimes, Bouches-du-Rhône, Var, éd. R. Favreau, J. Michaud, B. Mora, Paris, 1989, p. 84. (Musée Camarguais).

28. On garde néanmoins toujours au Moyen Âge l'idée que le $I^{\text {er }}$ janvier est le premier jour de l'an. Une inscription de Sainte-Geneviève de Paris de 1287 en rend compte : au mois de janvier, jour de l'an ». 


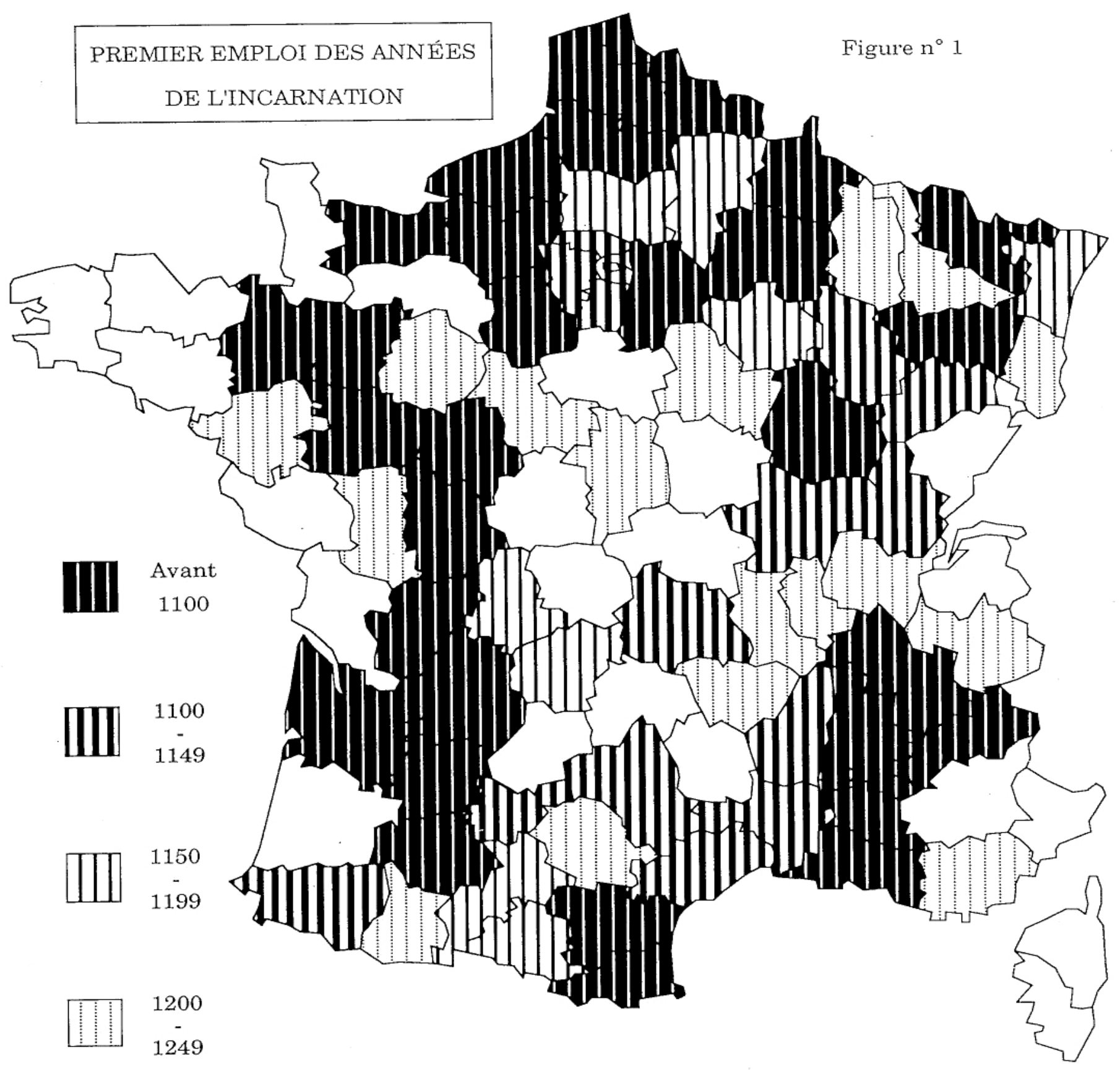


sion « anno ab Incarnatione domini (nostri) Jesu Christi », de 990 à 1216 , avec un exemple supplémentaire en 1337. Le français n'a pris que modérément le relais : "en l'an de l'Incarnation " de 1253 à 1312 et en 1360 , " an de l'Incarnation Nostre Seigneur Jesu Christ » de 1303 à 1329 et de 1395 à 1476 (quatre exemples). On trouve neuf inscriptions du nord de la Seine et de la Champagne datées de l' "anno incarnati Verbi (ou) Verbi incarnati ", de 1069 à 1204, puis sept dans la France du sud de 1219 à 1251, avec un exemple isolé en 1493. On note un « anni ab Incarnatione Christi » à Monestier-Allemont (Hautes-Alpes) en 1036, deux « anno Incarnacionis Christi " en 1177 et 1200 ou 1216 en Roussillon. La référence à l'Incarnation a ainsi été première du IX ${ }^{e}$ siècle au premier tiers du XIVe, avec quelques emplois sporadiques ultérieurs. L'emploi du mot Nativitas est peu fréquent et tardif, puisque tous les exemples, en mettant à part une inscription de 1094 à la cathédrale de Metz ${ }^{29}$, appartiennent au XIII ${ }^{e}$ siècle et, plus nombreux encore, aux deux siècles suivants ${ }^{30}$. C'est aussi aux XIV et $\mathrm{XV}^{\mathrm{e}}$ siècles qu'on relève les quatre exemples de " anno a Virginis partu (ou) Virginei partus ". Peut-être ces références à la Nativité et à l'Enfantement virginal sont-ils à mettre en rapport avec les progrès du culte marial.

L' «anno gratie » apparaît d'abord dans la France du sud, en 1121 à Montfrin (Gard), en 1200 à Vienne, en 1273 à Narbonne, et sous la forme française en 1233 à Saint-Léon-sur-Vézère (Dordogne), en 1325 et 1350 à Urville (Cantal). Pour la France du nord, les huit exemples de l'expression latine ont pour point de départ l'année 1218 («anno gracie » à Braine-sur-Vesle, Aisne); et, surtout, les exemples de l'expression française ${ }^{31}$, au nombre de plus de cinq cents, montrent la vogue de la formule dans la France du nord et de l'est, de la Normandie à la Bourgogne. Les exemples restent limités en France du sud et ne dépassent pas le XIII ${ }^{e}$ siècle, sauf à Urville (1325, 1350). Aucun exemple n'a été relevé en Alsace.

Mais la façon la plus courante de formuler la référence à l'ère chrétienne est de recourir à l'expression "anno Domini ", que l'on trouve dès les IXe et $\mathrm{X}^{\mathrm{e}}$ siècles (quatre exemples), au XI' $\mathrm{XI}^{\mathrm{e}}$ siècle (huit exemples), au XII' siècle (trente et un exemples), et surtout à partir du XIII' siècle, où les exemples atteignent presque le nombre de cinq cents. C'est même la seule formule

29. Xavier Barbiex de Montault, Les croix de plomb placées dans les tombeaux en manière de "pitacium ", dans Bulletin de la Société archéologique et historique du Limousin, t. 36, 1888, p. 23-49, à la p. 36-37 ; Gallia christiana, t. XIII, col. 735.

30. Trois exemples de « anno Nativitatis Christi " de 1210 à 1230 ou 1239 à Narbonne et à la Tour-sur-Orb en Languedoc, les autres exemples se rencontrant, sous cette forme avec « anno a Nativitate Domini » ou une autre expression, seulement à partir de 1380.

31. La forme habituelle est " an de grâce", mais on trouve aussi " l'an de grace Nostre Seigneur », « l'an de gracia ", "l'an de grasce », " en l'an di grace ", " en l'an de grace ». 
en Alsace à partir de 1214. "Anno Christi "n'a été employé qu'à partir du XIII' siècle. Deux exemples de son emploi se rencontrent à Saint-Vaast d'Arras, mais tous les autres se situent dans le sud-ouest, et en particulier en Roussillon. Après 1312, on ne remarque que des emplois isolés, à Perpignan en 1401, à Paris en 1413. On rencontre encore de nombreux " anno " ou « $a n$ ", sans autre précision, mais de façon moins fréquente, la référence chrétienne restant fortement majoritaire, quelle que soit l'expression retenue.

Des usages provinciaux apparaissent à travers l'emploi de certaines formules. Ainsi, c'est dans l'est, et principalement en Franche-Comté, à partir de 1290 et surtout au XIV siècle, que se trouve l'expression " l'an de notre Seigneur courant », qui fait écho au bel « an où le miliaire courait... » des actes, tandis que les expressions précédées de $s u b$ ("sub annis ", "sub annis Christi ", "sub anno Domini », "sub anno Incarnationis Domini ») sont localisées, du XI $\mathrm{XI}^{\mathbf{e}}$ au XIV siècle, en France du sud, à l'exception de l'exemple le plus récent, de 1388, à Saint-Martin-des-Champs de Paris (peut-être sous l'influence de Cluny dont il est prieuré). On relève quelques "anno salutis " aux $\mathrm{XIV}^{\mathrm{e}}$ et $\mathrm{XV}^{\mathrm{e}}$ siècles, tant au sud qu'au nord.

L'expression " anno dominice trabeacionis DCCCCLXXII" à Étoile-surRhône ne doit pas être comprise comme l'indication d'un usage de l'an de la Passion commençant en l'an 33 de l'ère chrétienne, car les indications complémentaires de calendes, férie et indiction correspondent bien à l'année $972^{32}$. A. Giry a souligné, à propos de l'emploi de la formule dans les chartes $\mathrm{du} \mathrm{XI} \mathrm{XI}^{\mathrm{e}}$ siècle, qu'il fallait faire dériver trabeatio de trabea (tunique) et non de trabs (poutre), et donc y voir un équivalent d'Incarnatio ${ }^{33}$ : l'hypothèse est étayée par un sermon de saint Fulgence de Ruspe, qui applique l'expression " trabea carnis inductus " à l'Incarnation ${ }^{34}$. La mention " anno a Passione Domini " se présente à Angers au XI $\mathrm{XI}^{\mathrm{e}}$ siècle ${ }^{35}$; et à Riom la formule " post Passionem anno Domini 1475 »36 doit en fait répondre à une année de l'Incarnation. Au XI' ou XIII' siècle, les chanoines de Saint-Front de Périgueux, composant une inscription pour conforter l'authenticité de leur saint patron, disciple prétendu de saint Pierre, mentionnèrent que Front

32. C.I.F.M., 16, p. 133-135.

33. A. Giry, Manuel..., p. 90.

34. Patr. lat., t. 65, col. 729 : « rex noster, trabea carnis indutus, de aula uteri virginalis egrediens, visitare dignatus est mundum ".

35. X. Barbier de Montault, Les croix de plomb..., p. 32-34 : au musée d'Angers, croix provenant de la Trinité d'Angers.

36. Docteur Roux, Note complémentaire pour les origines de Riom, dans Bulletin historique et scientifique de l'Auvergne, 1907, p. 191-197, fac-sim., h.-t. 
était mort en "l'an 42 après la Passion du Christ ", soit en 75 . On ne peut pour autant à proprement parler d'une ère de la Passion ${ }^{37}$.

L'ère d'Espagne, qui prend pour point de départ l'an 39 avant J.-C., peutêtre en souvenir de l'achèvement de la conquête romaine de l'Espagne, n'a que fort peu touché les inscriptions de la France, puisqu'on ne la trouve mentionnée qu'à deux reprises, en 989 à Sainte-Christie (Gers) où elle est le seul élément de datation, en 1141 à la cathédrale de Lescar où elle est donnée après l'année de l'Incarnation ${ }^{38}$.

Si l'on fait le calcul à partir des seize premiers volumes publiés du Corpus des inscriptions de la France médiévale, qui correspondent à la France $\mathrm{du}$ sud en sa majeure partie, soit quelque 2000 inscriptions, on constate qu'un peu plus de $72 \%$ des textes n'ont pas d'indication de l'année de l'Incarnation ou d'une année de règne, alors que près de $28 \%$ sont "datés ", et l'on peut noter que c'est seulement à partir du XIII siècle que la datation par l'année de l'Incarnation se généralise, puisque ces $28 \%$ se répartissent en $0,6 \%$ avant l'an mil, $1,55 \%$ au XI $\mathrm{XI}^{e}$ siècle, $5,7 \%$ au XII ${ }^{e}$, $20 \%$ au XIII ${ }^{e}$. Aux XIV et $X^{e}$ siècles, l'épigraphiste n'a plus habituellement à se préoccuper de "dater " les inscriptions, comme il doit le faire dans la généralité des cas avant le XII ${ }^{e}$ siècle ${ }^{39}$.

On notera comme une simple curiosité des références au sixième âge du monde, celui qui commençait avec l'avènement du Christ ${ }^{40}$. On le trouve indiqué à l'abbaye S. Stephano de Riba de Sil en 925, et à l'abbaye San Pelayo d'Oviedo en 1039.

La datation s'est faite aussi par chronogramme, en utilisant les lettres d'une phrase ou d'un vers qui correspondent à des chiffres romains. Cette manifestation de virtuosité se rencontre de bonne heure, car Victor H. Elbern en a donné de nombreux exemples dès la fin du VII" ${ }^{e}$ siècle, le premier étant l'inscription du calice de Tassilo à Kremsmünster, "Tassilo dux fortis, Livtpirc virga regalis »: en additionnant toutes les lettres correspondant à des

37. C.I.F.M., 5, Dordogne, Gironde, éd. R. Favreau, Bernadette Leplant, J. Michaud, Poitiers, 1979 , p. $40-42$.

38. C.I.F.M., 6, Gers, Landes, Lot-et-Garonne, Pyrénées-Atlantiques, éd. R. Favreau, B. Leplant, J. Michaud, Paris, 1981, p. 75 et 154-155.

39. Il ne sera pas fait état ici d'inscriptions hébraïques ou arabes qui ont leur système propre de datation. Une thèse de doctorat récente d'Anne Boulé sur l'Épigraphie florentine : les inscriptions commémoratives des $X I X^{e}-X X^{c}$ siècles (Université de Paris-Sorbonne, 1991), t. III, p. 262, cite une inscription de 1939 commémorant la fondation de l'Associazione Combattenti, datée de l'ère fasciste (1939-XVII).

40. Marie-Dominique Chenu, La théologie au douzième siècle, $2^{2}$ éd., Paris, 1966, p. 72 et suiv. 
chiffres, D, C, L, X, V, I, on dévoile l'année 781 41. Les chronogrammes sont plutôt employés en France aux XIV et XV siècles, ainsi pour la SainteChapelle de Bourges construite par Jean de Berry ${ }^{42}$ ou pour la cloche de la tour de l'Horloge à Paris ${ }^{43}$. On peut aboutir à un véritable rébus, comme le montre une curieuse inscription de l'hôtel de ville de Saint-Quentin en 1509 :

\author{
« D'UN MOUTON ET DE CINQ CHEVAUX \\ TOUTES LES TESTES PRENDREZ \\ ET A ICELLES SANS NULS TRAVAUX \\ LA QVEVE D'UN VEAV IOINDREZ \\ ET AU BOUT ADIOVSTREREZ \\ TOUS LES QUATRE PIEDS D'VNE CHATTE \\ RASSEMBLEZ VOUS APPRENDREZ \\ L'AN DE MA FAÇON ET SA DATE $\gg{ }^{44}$.
}

Il faut prendre les premières lettres (les têtes) de « mouton » et " chevaux ", soit $M$ et cinq fois $C$, $y$ joindre la dernière lettre (la queue) de "veau ", soit $\mathrm{V}$, et y ajouter les quatre unités des quatre pieds d'une chatte, ce qui correspond bien à 1509, MCCCCCVIIII.

Toutefois, le chronogramme a été beaucoup plus en vogue en Belgique, aux Pays-Bas, en Allemagne, en Autriche qu'en France.

2. Cycles et multiplication des indications chronologiques. - On a peu utilisé, dans les inscriptions médiévales, les périodes chronologiques. Les olympiades, introduites en Grèce par l'historien Timée (352-256 avant J.-C.),

41. Victor H. Elbern, Der eucharistiche Kelch im frühen Mittelalter, dans Zeitschrift des deutschen Vereins für Kunstwissenschaft, t. 17, 1963, p. 1-76 et 117-188, aux p. 7-10.

42. P. des Chaumes, L'inscription numérale de la Sainte-Chapelle de Bourges, dans Mémoires de la Société historique, littéraire et scientifique du Cher, 4e sér., t. 37, 1928-1929, p. 163-172; Maurice de Laugardière, Le cryptogramme de la Sainte-Chapelle, ibid., p. 173-176.

43. Léon Mirot, Date de la cloche d'argent du palais à Paris, dans Bulletin de la Société nationale des antiquaires de France, 1920, p. 193-195; Roger Rodière, Rectification de la lecture du chronogramme de la cloche du palais à Paris, ibid., 1927, p. 161-162. Pour le XVe siècle : Charles Samaran, Chronogrammes du XV siècle, ibid., 1934, p. 173; id., Note complémentaire sur quelques chronogrammes dans les manuscrits du Xve siècle, dans Mémoires de la Société nationale des antiquaires de France, t. 80 (= 8e série, t. 10), 1937, p. 249-250; Jean Babelon, Chronogrammes dans les médailles du XV siècle, ibid., p. 247-248. Voir aussi James Hilton, Chronograms 5000 and more in number excepted of various authors and collected at many places, Londres, 1882; André Lépine, 400 chronogrammes curieux, Cerfontaine, 1987 (depuis 1112).

44. François de Guilhermy, Archéologie lä̈que, dans Annales archéologiques, t. 19, 1859, p. 253-277, à la p. 262 . 
parfois citées avec affectation d'érudition dans quelques chartes des $X^{\text {e }}$ et $\mathrm{XI}^{e}$ siècles, sont ignorées des inscriptions médiévales en France. Une épitaphe de Filatierra de 752 qui en fait état est de toute façon une exception ${ }^{45}$. En revanche, on a pratiqué, rarement mais régulièrement, le système de l'indiction, dont une novelle de Justinien rendit l'emploi obligatoire dans les actes ${ }^{46}$. Mais cette pratique n'a pas été générale, ce qui s'observe dès les inscriptions chrétiennes, puisque, à Trèves, on ne trouve aucune mention d'indiction ${ }^{47}$, alors que la Viennoise du nord en fournit vingt-six exemples précis, auxquels on ajoutera dix-huit inscriptions où l'indiction a pu être gravée ${ }^{48}$. Les premiers exemples dans le sud-est en remontent à la fin du $\mathrm{V}^{\mathrm{e}}$ siècle $(487,491,495 \ldots)$ et l'emploi n'en devint courant que vers 536-538 ${ }^{49}$. À la suite des inscriptions chrétiennes, on rencontre l'indiction dans les inscriptions médiévales de France du IX ${ }^{e}$ au $\mathrm{XII}^{\mathrm{e}}$ siècle, avec deux exemples ultérieurs, l'un en 1362 à Maisonnais-surTardoire en Limousin, l'autre en 1419 en Berry 50.

La plus grande fréquence d'emploi de l'indiction s'observe à l'est du Rhône (fig. 2). Aucun exemple ne peut être cité pour le Centre-ouest, l'Auvergne, les pays de Loire, l'île-de-France, la Picardie, la Champagne. La Bourgogne n'apporte qu'une inscription, à Saint-Bénigne de Dijon en 1103. Souvent la mention de l'indiction va de pair avec d'autres indications chronologiques qui tendraient à montrer que les auteurs avaient des tables à leur disposition.

À Monetier-Allemont (Hautes-Alpes) en 1036, on relève, avec l'an de l'Incarnation, l'indiction, l'épacte, le concurrent, les calendes, la lune, la férie ${ }^{51}$; à Saint-Macaire (Gironde) en 1039, l'Incarnation, l'épacte, l'indiction, le concurrent, le jour du mois, le jour de la lune ${ }^{52}$; à Saint-Amandles-Eaux (Nord) en 1088, l'an de l'Incarnation, l'indiction, l'épacte, le concurrent, le terme pascal, le calendrier romain ${ }^{53}$; à Saint-Melaine de Rennes

45. Arturo Carlo Quintavalle, La strada romea, Parme, 1975, p. 163.

46. F. Descombes, Recueil des inscriptions chrétiennes..., XV, p. 58.

47. N. Gauthier, Recueil des inscriptions chrétiennes..., I, p. 48.

48. F. Descombes, Recueil des inscriptions chrétiennes..., XV, p. 48.

49. Ibid., p. 58 : en 487 à Saint-Thomé (Ardèche), fin 491 en Viennoise du nord, 495 à Arles.

50. Épitaphe d'Arnulfe Belin, trésorier de la Sainte-Chapelle de Bourges, mort en 1436, enterré dans la Maison-Dieu d'Aubignac (Parnac, Indre) qu'il avait fondée et dotée " anno circiter 1419» (Gallia christiana, t. II, col. 115).

51. C.I.F.M., 16, p. 39.

52. C.I.F.M., 5, p. 121.

53. Louis Serbat, Inscriptions funéraires de recluses à l'abbaye de Saint-Amand (Nord), dans Mémoires de la Société nationale des antiquaires de France, t. 72 (= $8^{\mathrm{e}}$ sér., t. 1), 1912, p. 193-224, ill. 


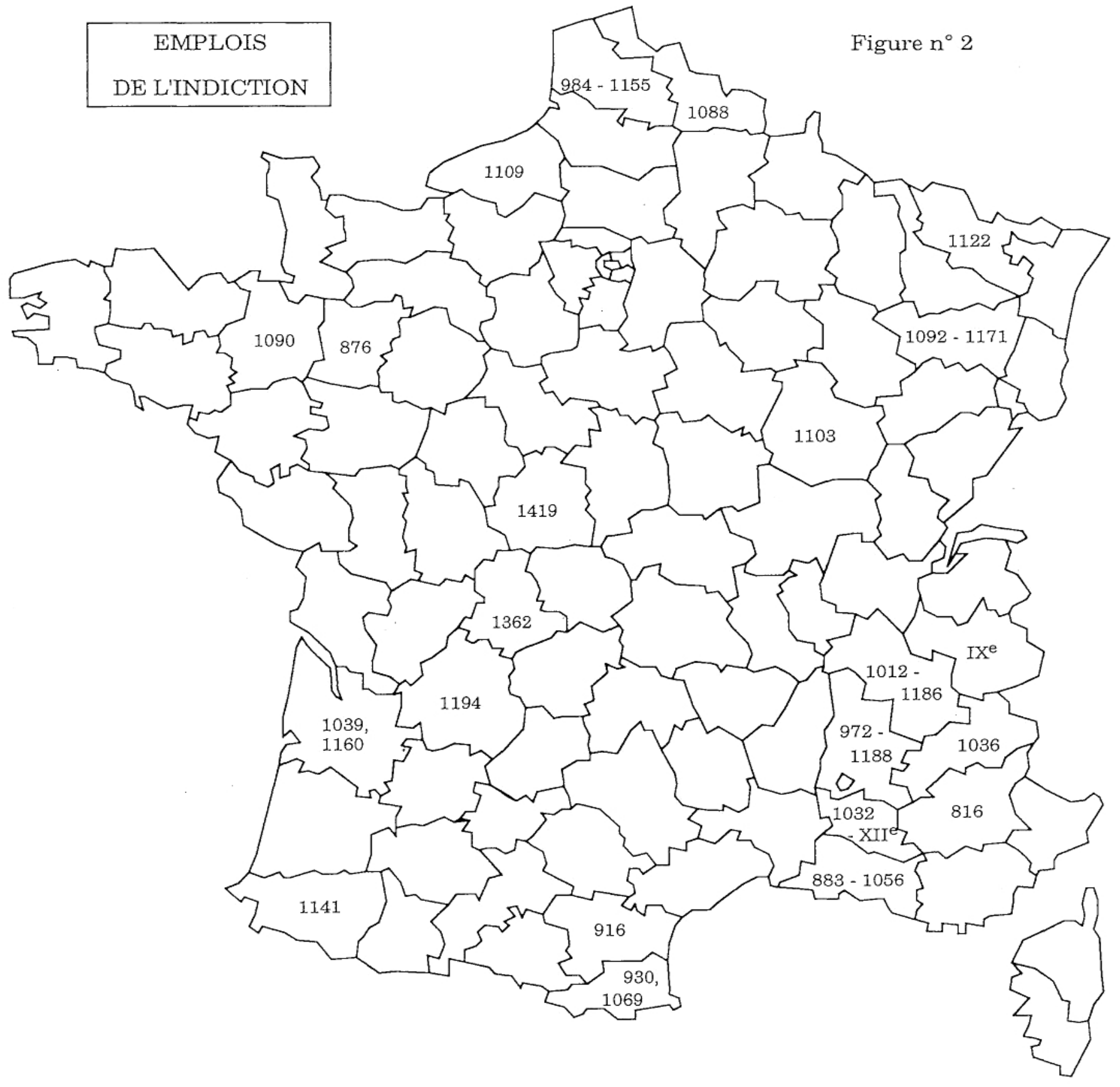


en 1090, l'an de l'Incarnation, l'indiction, le concurrent, les ides ${ }^{54}$, etc. Le record de précisions chronologiques figure sans doute dans cette inscription de dédicace de Saint-Martin de Limeuil (Dordogne), en 1194, qui cite l'Incarnation, l'indiction, le concurrent, l'épacte, le jour de la semaine, le jour des calendes, la lunaison, et donne les noms des diacres de l'église, du pape, du roi de France, du duc d'Aquitaine, du comte de Périgord, de l'archevêque de Bordeaux ${ }^{55}$, multiples mentions que l'on retrouve dans une charte du cartulaire de la proche abbaye de Cadouin de 1189. Certaines abbayes ont affectionné ces précisions chronologiques, ainsi de celle de Remiremont (Vosges), avec, de 1092 à 1182, huit inscriptions à mentions chronologiques multiples, dont cinq avec l'indiction.

Les mentions d'indiction sont d'une utilité très faible pour une datation, car il est exceptionnel que ce soit la seule mention chronologique, et par ailleurs il faudrait être assuré du point de départ du comput, $I^{\text {er }}$ septembre, 24 septembre, 25 décembre ou $I^{\text {er }}$ janvier ${ }^{56}$. $\Pi$ y a des variations dans le calcul dès les inscriptions chrétiennes; il y en a aussi dans les inscriptions " médiévales ". Pour y voir plus clair ici, il faudrait utiliser conjointement chartes, chroniques et inscriptions. Celles-ci, d'ailleurs, ne fournissent, pour les $\mathrm{IX}^{\mathrm{e}}-\mathrm{XII}^{\mathrm{e}}$ siècles, qu'une quarantaine d'exemples, c'est-à-dire à peu près autant que les seules inscriptions chrétiennes de la Viennoise du nord, les inscriptions médiévales offrant six exemples d'emploi de l'indiction pour Vienne aux $\mathrm{XI}^{e}$ et $\mathrm{XII}^{\mathrm{e}}$ siècles. Étudiant la datation des inscriptions romaines, I. Kajanto note quelques exemples tardifs d'emploi de l'indiction encore au $\mathrm{XV}^{\mathrm{e}}$ siècle, et parle d'une " considérable fréquence » de l'indiction dans l'épigraphie médiévale ${ }^{57}$. On ne peut dire que ce soit le cas pour la France.

3. Le calendrier romain des jours. - Il était plus important, au Moyen Âge, de connaître le jour du mois que l'année, notamment parce que la connaissance du jour était indispensable pour célébrer la mémoire du défunt chaque année ou pour fêter l'anniversaire de la dédicace d'une église. Plusieurs systèmes de datation se sont ici succédé, en se chevauchant pour une grande partie du temps, calendrier romain, quantième du mois, mention de fête religieuse.

L'emploi du calendrier romain est l'usage le plus fréquent dans les inscriptions les plus anciennes. Il se raréfie progressivement à partir surtout de la seconde moitié du XII ${ }^{e}$ siècle et n'est guère employé après le premier

54. X. Barbier de Montault, Les croix de plomb..., p. 35-36.

55. C.I.F.M., 5, p. 21-23.

56. A. Giry, Manuel..., p. 98-99.

57. I. Kajanto, Dating in the Latin inscriptions..., p. 46. 
tiers du XIV siècle, même si on trouve des utilisations sporadiques au $\mathrm{XV}^{\mathrm{e}}$ siècle dans un certain nombre de départements après une longue interruption, vogue nouvelle due à l'humanisme de la Renaissance (fig. 3a-3b). Si l'on ne tient pas compte d'emplois isolés après une série continue, ce qui se présente, par rapport à ce qui suit, dans une quinzaine de cas, on peut dégager l'évolution suivante :

- Dans douze départements, il n'y a aucun exemple d'utilisation du calendrier romain. Ce ne sont que des départements pauvres en inscriptions, la seule surprise étant de trouver ici la Meurthe-et-Moselle.

- Dans douze départements répartis dans toute la France, l'emploi du calendrier romain ne dépasse pas le XII ${ }^{\mathrm{e}}$ siècle.

- Dans vingt-six départements, on n'utilise plus le calendrier romain, sauf un cas isolé tardif dans trois départements, après le XIIIe siècle.

- Dans vingt-quatre départements, toujours sous la réserve de quelques exemples isolés du XV $\mathrm{XV}^{\mathrm{e}}$ siècle, l'emploi du calendrier romain ne se trouve plus après 1334 .

- Onze nouveaux départements ont encore des exemples de l'utilisation du calendrier romain dans les deux derniers tiers du XIV siècle.

- Dans dix départements, enfin, on peut considérer que le calendrier romain s'est maintenu de façon continue jusqu'au $\mathrm{XV}^{\mathbf{e}}$ siècle.

Si l'on essaie de repérer des répartitions géographiques, on voit que les sept départements actuels de la région parisienne ne connaissent plus le calendrier romain après 1301, sauf un exemple de 1341 en Val-de-Marne; dans la France du nord et la Champagne, si l'on écarte trois exemples isolés tardifs, l'emploi ne dépasse pas 1332. C'est en Bourgogne et Franche-Comté, en Alsace aussi sans doute, que le calendrier romain est le plus longtemps utilisé. Il faut y ajouter le Roussillon jusqu'en 1425. Lorsqu'on considère le nombre des exemples fournis par chaque département, on peut dire que c'est dans la France du sud-est et du sud-ouest qu'on a eu le plus souvent recours à ce mode de datation, avec cent trente-trois exemples pour les Pyrénées-Orientales, cent sept pour l'Isère, soixante et onze pour la HauteGaronne. Il est vrai que ce sont des départements où les inscriptions médiévales sont particulièrement nombreuses.

Le tableau présenté par I. Kajanto sur l'emploi respectif du calendrier romain et de la façon moderne de donner le quantième du mois à Rome montre que, malgré la fidélité de la papauté au calendrier classique, c'est à partir de la seconde moitié du XIII' siècle que le calendrier moderne l'emporte, avec une prépondérance écrasante, $95 \%$ ou plus de 1301 à 1450, le calendrier romain trouvant une seconde faveur au moment de la Renaissance, jusqu'à figurer dans $40 \%$ des 321 inscriptions comprises entre 1500 







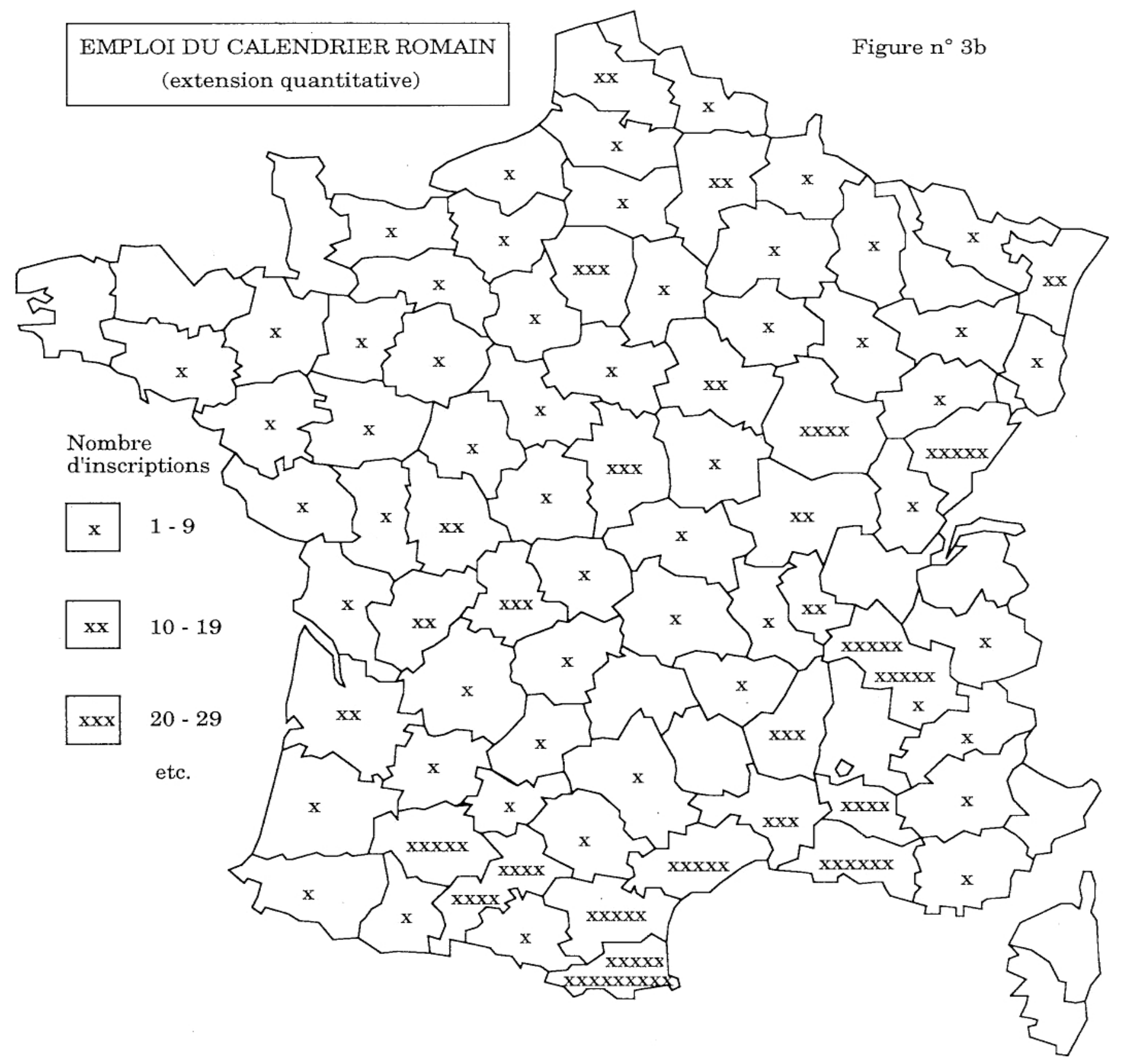


et $1527^{\mathrm{s}}$. Cette reprise de l'usage du calendrier romain s'observe aussi en France au XVe siècle, mais avec des exemples beaucoup moins nombreux, sans doute dans la volonté, comme à Rome ${ }^{59}$, de faire revivre les usages de l'Antiquité devenue une nouvelle fois le modèle. À Rome, cette réapparition de la datation classique s'observe à partir de 1430 seulement. En France, la généralisation de cette réapparition s'effectue à partir de 1460 , mais on peut citer cinq ou six exemples pour les années 1410, 1420, 1430.

4. Nouvelles notations : quantièmes et féries. - I. Kajanto a relevé les débuts de l'emploi de la façon moderne de donner le quantième du mois ${ }^{60}$. Le premier exemple serait représenté par une inscription à Terracina en $345^{61}$, les suivants se trouvant en 506 à Nola et à Capoue en 517, avec quelques autres aux $\mathrm{VII}^{\mathrm{e}}$ et $\mathrm{VIII}^{e}$ siècles ${ }^{62}$. Avec Charlemagne, le système classique aurait repris une prépondérance absolue. La datation moderne redémarre à Rome dans les années 980 . I. Kajanto en note onze exemples à Rome au XIe siècle, et un seul au XII $\mathrm{XI}^{\mathrm{e}}$, trois pour la première moitié du XIII et déjà quinze sur un total de vingt-trois inscriptions pour la seconde moitié du même siècle.

Du côté des inscriptions médiévales françaises, on dispose d'un exemple de notation moderne dans l'épitaphe métrique de Folcuin, évêque de Thérouanne, mort en 855, "die quarto decimo decembris "63. Ensuite, on trouve l'épitaphe de la fondatrice de l'abbaye de Bernay, Judith Conan, morte en 1017, "decimo septimo junii ${ }^{64}$; mais il faudrait étudier de près le document, car le formulaire employé n'est pas celui $\mathrm{du} \mathrm{XI}^{\mathrm{e}}$ siècle, la transcription du $i$ final long par un $y$ dénote une copie, et l'on pourrait plutôt se trouver devant un texte fait ou refait plus tard. La translation des reliques de saint Florent de Roye à Saumur est datée par un " transtulit anno Domini $M X X X V$ die mai $X X V$ ", dans lequel on a vu l'inscription qu'on lisait sur

58. Ibid., p. 48.

59. Ibid., p. 50; l'auteur note aussi à Rome cette réapparition d'une datation "classique " au XV' siècle, à partir de 1430 .

60. Ibid., p. $46-48$.

61. Ibid., p. 46-48; Ernest Diehl, Inscriptiones latinae christianae veteres, II, Berlin, 1927, $n^{\circ} 4422$, p. 419. L'inscription a été d'abord publiée dans le Corpus inscriptionum latinarum, t. X, Berlin, 1883, pars posterior, p. 1015; " die VI menso junii », ibid., t. X, pars prior, Inscriptiones Bruttiorum, Lucaniae, Campaniae, addition au ${ }^{\circ} 6420$, p. 635.

62. N. Gauthier, Recueil des inscriptions chrétiennes..., I, $\mathrm{n}^{\circ} 147, \mathrm{p} .381-383, \mathrm{vI}^{\mathrm{e}}$ ou VIII $^{e}$ siècle, nécropole de Saint-Paulin-Saint-Maximin de Trèves.

63. Vita Folquini episcopi Morinensis, auctore Folquino abbate Laubiensi, éd. Oswald HolderEgger, dans M.G.H., SS, t. XV-1 (Hanovre, 1887), p. 430.

64. Léon Métayer-Masselin, Mémoire sur une ancienne crosse trouvée à Bernay, dans Bulletin monumental, t. 26, 1860, p. 794-802, à la p. 794, n. 1 . 
la châsse du saint ${ }^{65}$. On ne peut mettre en doute l'épitaphe versifiée de la reine Mathilde, épouse de Guillaume le Conquérant, dans l'église abbatiale de la Trinité de Caen, qui porte que la reine mourut « in prima mensis, post primam, luce novembris $" 66$. Les exemples ne commencent à se suivre de façon continue qu'à partir de la seconde moitié du XII siècle : en 1158 à Notre-Dame de Chaage à Meaux, en 1160 à l'abbaye d'Igny en Champagne, en 1169 à Saint-Étienne de Périgueux, en 1183 à Saint-Nicolas d'Arras, en 1189 à Saint-Jacques de Strasbourg. Tous ces exemples, sauf celui de Périgueux, appartiennent à la moitié nord de la France. L'épitaphe de Périgueux concerne l'évêque Jean d'Asside, qui était d'origine poitevine, et elle est l'œuvre de Constantin de Jarnac ${ }^{67}$.

$\mathrm{Au}$ XIII ${ }^{\mathrm{e}}$ siècle, la notation moderne des jours se rencontre en Norman-


principalement à partir de la seconde moitié du siècle, puisque l'on ne possède que deux exemples pour sa première moitié ${ }^{68}$. Dans le sud-est, ce mode de datation se répand au XIV siècle, après deux exemples isolés en 1270, à Villecroze (Var) ${ }^{69}$ et à l'abbaye de Hautecombe (Savoie). Pour le Languedoc-Roussillon, il en est de même, mais avec une exception à Nîmes qui offre quatre emplois de cette datation au XIII ${ }^{e}$ siècle, en 1203,1215 , 1241 et 1298. Dans la région Midi-Pyrénées, un exemple de 1280 à Toulouse est isolé, les autres exemples ne commençant qu'au XIV ${ }^{e}$ siècle. Dans la France centrale, deux exemples en Limousin, un dans l'Allier, pour les années 1250 et 1260 , sont des précurseurs d'un mode de datation qui, mis à part deux exemples en Limousin en 1331 et 1335 , ne se retrouve qu'à partir de 1360.

La datation par le quantième du mois est surtout accompagnée du mot dies. Moins souvent, elle détermine l'emploi conjoint des mots dies et mensis. L'emploi du seul mensis est rare. Les expressions n'indiquant ni dies ni mensis sont plus fréquentes dans la France de l'est que dans la France du nord et en Normandie, comme on peut le déduire d'un comptage entre-

65. Paul Marchegay, Communication d'un fragment..., p. 476, n. 1, et 481-482.

66. A. de Caumont, Statistique monumentale..., p. 30-31; Arthur Kingsley Porter, Medieval architecture, its origines and development, t. I, New Haven, 1912, p. 286; Michel de Boüard, Histoire de la Normandie, Toulouse, 1970, p. 112-123, pl. h.-t.

67. C.I.F.M., 5, p. 31-34.

68. Pour la première moitié du XIII ${ }^{e}$ siècle, un exemple pour la Seine en 1208 , un pour l'Oise en 1219.

69. Plutôt « 1270,8 mai » que « 1278 , mai ». 
pris pour les douze départements où les exemples sont les plus nombreux (tabl. 1).

\begin{tabular}{|l|c|c|c|c|}
\hline & $\begin{array}{c}\text { Avec dies et } \\
\text { mensis }\end{array}$ & $\begin{array}{c}\text { Avec dies } \\
\text { seul }\end{array}$ & $\begin{array}{c}\text { Avec mensis } \\
\text { seul }\end{array}$ & $\begin{array}{c}\text { Sans dies ni } \\
\text { mensis }\end{array}$ \\
\hline Aube & 3 & 14 & - & 12 \\
\hline Calvados & 4 & 20 & 1 & 1 \\
\hline Côte-d'Or & 39 & 77 & 7 & 27 \\
\hline Doubs & 25 & 24 & 10 & 19 \\
\hline Jura & 6 & 15 & 1 & 9 \\
\hline Marne & 32 & 38 & 3 & 8 \\
\hline Nord & 11 & 9 & - & 4 \\
\hline Oise & 8 & 22 & 3 & 4 \\
\hline Pas-de-Calais & 35 & 62 & 3 & 10 \\
\hline Seine & 30 & 48 & 4 & 4 \\
\hline Seine-Saint-Denis & 10 & 14 & 3 & 4 \\
\hline Seine-Maritime & 1 & 23 & & 4 \\
\hline
\end{tabular}

Tableau $n^{\circ}$ 1. - Modes d'expression du quantième dans les inscriptions de douze départements.

Ce mode nouveau de datation a été reçu moins facilement dans la France du sud, dans la mesure où il imposait un calcul différent de celui du calendrier romain, où l'on compte les jours à rebours. On trouve en Gascogne trois exemples où l'on a donné le quantième à partir de la fin du mois, en suivant l'usage du mos bononiensis des rédacteurs d'actes : à l'abbaye de Flaran en 1305, "VII die exitus septembris ", et deux fois à Auch en 1333 et 1348 avec le même mot exitus; on peut y joindre un exemple à Blagnac en Toulousain en 1283. En sens inverse, toujours dans le sud-ouest, en 1321 à deux reprises à Toulouse, en 1333 à Saint-Sardos, on a jugé bon d'indi- 
quer que les jours étaient comptés à partir de l'entrée du mois, introitus mensis. On trouve un exemple similaire dans la moitié nord de la France, à Saint-Nicaise de Reims, en l'an de grâce 1298 " deux jours en la fin de juillet ".

L'emploi de la férie n'a eu qu'un succès médiocre : un exemple au VHI $\mathrm{IX}^{\mathrm{e}}$ siècle, un au $\mathrm{X}^{\mathrm{e}}$, un au $\mathrm{XI}^{\mathrm{e}}$, neuf au XII ${ }^{e}$, quatre au XII" ${ }^{e}$, deux au XIVe siècle, bien qu'il ait été promu par l'Église pour détrôner la façon romaine, païenne, de nommer les jours de la semaine. Comme dans la langue courante, l'exception est constituée par le dimanche, " jour du Seigneur ", qui a tôt remplacé le " jour du soleil » : des exemples sont signalés à Rome en 404, à Trèves en 409, à Villeneuve-lès-Avignon en 586-587. À Rome aussi, I. Kajanto ne relève que trois emplois de la férie dans des inscriptions, en 1248, 1263, 1504, 70 .

5. Le calendrier liturgique. - La datation par une année comptée à partir de l'Incarnation a été complétée par un « style " de changement d'année qui intervenait au moment d'une fête religieuse, Annonciation, Nativité, Pâques, voire Circoncision. Il y a donc une certaine logique, dans la chrétienté médiévale, à donner aussi le jour du mois à partir d'une fête religieuse, d'autant plus que clercs séculiers ou réguliers avaient un contact constant avec les livres liturgiques, qui le plus souvent comportaient des calendriers liturgiques avec les fêtes de l'Église universelle et avec les fêtes des saints vénérés localement.

Sur le fût de deux colonnes de marbre près de l'église Sainte-Marie de Carmona, aux environs de Séville, se trouvait gravé un calendrier liturgique, dès le début du VI ou même la fin du V $V^{e}$ siècle; l'Espagne conserve encore un fragment épigraphique de martyrologe, à Italica en Bétique, du VI ${ }^{e}$ ou VII siècle. À San Giovanni Maggiore à Naples, on a trouvé, lors d'une restauration, deux longues tables de marbre, que l'on date de 840 ou 850 , comportant un calendrier complet, avec par ailleurs la façon moderne de donner le quantième du mois. Un calendrier était peint sur le mur du cloître du monastère Sainte-Marie de l'Aventin à Rome, au milieu du XIe siècle; un autre subsiste encore, peint sur la partie droite de la nef de l'église des Quatre-Saints-Couronnés à Rome, dans la seconde moitié du XIII ${ }^{e}$ siècle. On rappellera aussi les deux tables gravées, de saints et de saintes, du $\mathrm{XI}^{\mathrm{e}}$ siècle, fixées de chaque côté du vestibule de Saint-Sylvestre-in-Capite à Rome ${ }^{71}$. En France, on a trouvé vers 1917 à l'évêché d'Évreux, sous

70. I. Kajanto, Dating in the Latin inscriptions..., p. 58.

71. R. Favreau, L'épigraphie comme source pour la liturgie, dans Vom Quellenwert der Inschriften, Vorträge und Berichte der Fachtagung, Esslingen, 1990, hrsg. Renate NeumullersKlauser, Heidelberg, 1992, p. 65-137, aux p. 68-69. 
badigeon, un calendrier liturgique peint, en belle gothique du XIII siècle $^{72}$, et il faut rappeler le très curieux document donnant une table perpétuelle du jour de Pâques, gravé sur marbre au XII' siècle, conservé dans l'église Saint-Étienne de Périgueux, ou le calendrier donnant la clé des fêtes mobiles qui se trouvait à l'abbaye de Stürzelbronn, dans le diocèse de Metz, au XII ${ }^{e}$ ou au XIII' siècle $^{73}$.

On trouve les premiers exemples de datation par référence à la fête d'un saint à Briord aux VI ${ }^{e}-V_{I I}^{e}$ siècles (la Saint-Martin) ${ }^{74}$, à Tarragone dans une inscription de l'époque wisigothique (le jour de la "déposition de saint Pierre ", 22 février, fête de la chaire de saint Pierre) ${ }^{75}$. Aucun exemple identique ne se présente en France pour la période carolingienne. Le chroniqueur Adémar de Chabannes nous a transmis l'épitaphe du comte d'Angoulême Guillaume II, décédé la veille des Rameaux en 1028; du fait sans doute de la nouveauté de ce mode de datation, l'auteur de l'épitaphe a, en même temps, donné le jour à partir du calendrier romain : «VIII idus aprilis, vigilia Osanna " ${ }^{76}$. L'épitaphe versifiée de l'archidiacre Fulbert à Saint-Ouen de Rouen, vers 1128, connue grâce à Orderic Vital, situe la date du décès quatre jours avant Noël 77 .

Une datation renvoyant à la fête d'un saint se présente dans l'épitaphe de Stefania à Toulouges (Pyrénées-Orientales), « in die sancti Martini ». L'inscription est en très mauvais état de conservation, l'écriture très maladroite semble reporter au $\mathrm{XI}^{e}$ siècle, mais le tracé redoublé du $A$ dans le mot $M a r$ tini reporterait plutôt au $\mathrm{XII}^{\mathrm{e}}$ siècle, le redoublement de trait s'observant, dans les inscriptions de la France du sud, dans la première (Laguenne en Corrèze) et surtout la seconde moitié du XIre siècle ${ }^{78}$. Une des épitaphes

72. Indication donnée dans le Bulletin archéologique de la Société archéologique du Tarnet-Garonne, t. 45, 1917, p. 135-136.

73. R. Favreau, L'épigraphie comme source..., p. 67-68. Un calendrier pascal pour les années 532-626 se trouvait aussi dans le vestibule de la cathédrale de Ravenne.

74. René Aigrain, Manuel d'épigraphie chrétienne, t. I, Paris, 1912, n²25, p. 103.

75. José Vivès, Inscripciones cristianas de la España romana y visigoda, Barcelone, 1969, n 199 , p. 63-64.

76. Adémar de Chabannes, Chronique, éd. Jules Chavanon, Paris, 1897 (Collection de textes pour servir à l'étude et à l'enseignement de l'histoire), p. 165-166; C.I.F.M., 3, Charente, Charente-Maritime, Deux-Sèvres, éd. R. Favreau, J. Michaud, Poitiers, 1977, p. 16-17.

77. The ecclesiastical history of Orderic Vitalis, t. IV, Books VII and VIII, éd. Marjorie Chibnall, Oxford, 1973 (Oxford medieval texts), p. 310.

78. C.I.F.M., 11, Pyrénées-Orientales, éd. R. Favreau, J. Michaud, B. Mora, Paris, 1986, $\mathrm{n}^{\circ}$ 136, p. 159-160. Le caractère très fruste des inscriptions de dédicace de Roullet (Charente) ou Peyrusse-Grande (Gers), où on trouve ce redoublement de trait, ne permet pas de dater ces textes avec certitude entre le XIe et le XIr siècle. J'ai écarté une inscription disparue datée d'environ 1007 à Saint-Bénigne de Dijon, « in festo sanctae Scolasticae ", parce qu'il est dit qu'on y voyait la figure du défunt avec son armure et une lance. 
versifiées, composées pour Boson, quatrième abbé du Bec-Hellouin, mort en 1136, fait référence à la Nativité de Jean Baptiste, "ortus Baptiste colitur $\gg 79$.

Le vrai départ de ce mode de datation se situe dans la seconde moitié du XII siècle : "vigilia beati Andreae » au musée de Narbonne en $1162^{80}$; " in depositione sancti Martini " sur une croix de plomb à Remiremont en $1182^{81}$; "crastina lux rapit hunc assumta Matre Potentis " à Elne en $1186{ }^{32}$; " in festo Eulalie " au cimetière Saint-Barthélemi de Montpellier en 1192 83; " bis oriente die sancti post festa Mathiae " vers 1193 à SaintNicaise de Reims ${ }^{84}$; " vigilia Purificacio sancta Maria " au XII ${ }^{e}$ siècle à la chapelle Saint-Pierre de Bagnols (Vaucluse) ${ }^{85}$. Les inscriptions de Courgenay (Yonne) en 1204, d'Égligny (Seine-et-Marne) en 1205 sont en français et les dessins qui en sont conservés par la collection Gaignières présentent des onciales fermées fort improbables à cette date-là. Ce mode de datation se répand, comme dans les actes, à partir du deuxième et surtout du troisième tiers $\mathrm{du} \mathrm{XIm}^{\mathrm{e}}$ siècle. Dans un nombre notable de cas, il marque un temps d'arrêt au milieu du XIV e, pour connaître une reprise au XVe siècle (fig. 4).

Si l'on examine la répartition géographique de l'emploi de la datation par une fête religieuse, on constate qu'il existe une différence sensible entre la France du sud et la France du nord. Dans la moitié méridionale du pays, de la Sèvre niortaise à Lyon et à la Savoie, c'est-à-dire presque exactement dans les pays de langue d'oc, cette datation est employée plus tôt qu'au nord, mais aussi plus rarement, et moins longtemps. Dans tous les exemples cités ci-dessus, antérieurs à 1200 , seules deux inscriptions représentent la France du nord, celles de Remiremont et de Reims. Sur trente-huit départements concernés, moins riches il est vrai en inscriptions des XTVe et $\mathrm{XV}^{e}$ siècles que les pays de la France du nord, dix-sept ne connaissent pas ce mode

79. Léopold Delisle, Notice sur vingt manuscrits du Vatican, dans Bibliothèque de l'École des chartes, t. 37, 1876, p. 471-527, aux p. 519-527 : "XX, Chronique du Bec, no 499 du fonds de la Reine "; Patr. lat., t. 150, col. 732.

80. C.I.F.M., 12, Aude, Hérault, éd. R. Favreau, J. Michaud, B. Mora, Paris, 1988, p. $60-61$.

81. Paul Chevreux, Les croix de plomb dites croix d'absolution de la région vosgienne, dans Bulletin archéologique du Comité des travaux historiques et scientifiques, 1904, p. 391-408, à la p. 4.02 , pl. XLI, no 1 .

82. C.I.F.M., 12, Paris, 1988, p. 60-61.

83. Ibid., p. 146-147.

84. Gallia christiana, t. IX, col. 213; abbé Midoux, L'ancienne église Saint-Nicaise de Reims, dans Bulletin monumental, t. 85, 1926, p. I17-152, aux p. 150-151.

85. C.I.F.M., 13, Gard, Lozère, Vaucluse, éd. R. Favreau, J. Michaud, B. Mora, Paris, 1988, p. 202. 


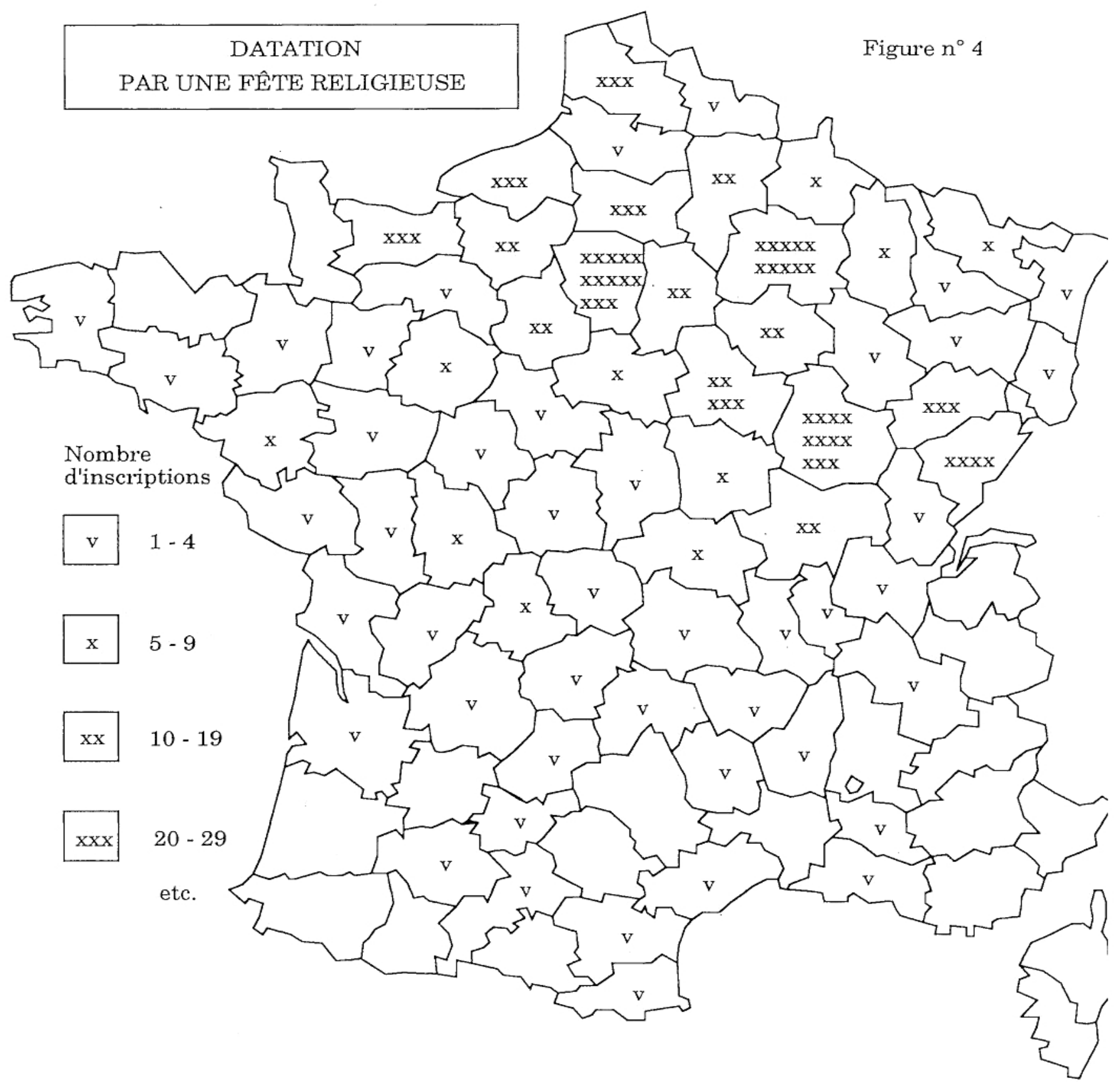


de datation. Celui-ci n'est plus utilisé après 1336, sauf un exemple en 1404 à Carpentras (Chaire de saint Pierre), un autre en 1409 à Clermont-Ferrand (Sainte-Lucie), et un dernier en 1493 à Vienne (Assomption de Marie). Ce système est particulièrement rare à l'est du Rhône où il n'est pratiqué que dans trois départements sur dix. Encore faut-il noter qu'on ne relève qu'un seul exemple pour les Bouches-du-Rhône, à Marseille en 1236, "fesítivìtate omnium sanctorum " (inscription du musée Borély, de provenance inconnue) ${ }^{86}$. C'est bien dans la moitié nord de la France, en particulier en Bourgogne et Franche-Comté, Champagne, Ille-de-France, Normandie, Beauvaisis et Artois que la datation à partir d'une fête religieuse a été le plus souvent employée. On peut même préciser que le temps fort de cet emploi se situe à la fin du XIÜ siècle et dans la première moitié du XIV ${ }^{e}$ siècle. Si l'on retient les douze départements où les exemples sont les plus nombreux, on totalise ainsi $22 \%$ des exemples avant $1300,54,6 \%$ pour la première moitié du XIV $, 11,6 \%$ pour la seconde moitié, $7 \%$ pour la première moitié du $\mathrm{XV}^{\mathrm{e}}, 4,5 \%$ pour la seconde moitié (tabl. 2).

Ce mode de datation permettrait d'établir une carte hagiographique des quatre-vingt-neuf saints et saintes qui y sont évoqués. On notera seulement ici que les saints auxquels les inscriptions se réfèrent le plus souvent sont, en ordre décroissant, Martin, Jean Baptiste, Madeleine, Pierre, André, Luc, Matthieu, Michel, Nicolas, Denis, etc. Pour les fêtes de la Vierge, les plus souvent citées sont la Nativité et l'Assomption, à égalité, puis la Purification et l'Annonciation, les renvois à la Conception étant nettement en retrait.

6. Notations chronologiques diverses. - La datation en référence à une année de jubilé représente un cas particulier, dont il n'a pas été tenu compte ci-dessus. Le domaine français en offre quatre exemples, chaque fois complétés par l'année de l'Incarnation, trois en référence au jubilé de 1400 , à Toul, Avignon et Limoges, et un en référence au jubilé de 1450, à Villefranche-de-Rouergue ${ }^{87}$.

Les épitaphes contiennent un certain nombre de mentions d'heure du décès. De façon plus générale, il fallait pouvoir suivre les heures de la journée, notamment pour les moines et la récitation des heures liturgiques, jusqu'à ce que l'apparition des horloges au XIV siècle ne vienne révolutionner les pratiques. En dehors des cadrans solaires, qu'on trouve en particulier en Angleterre et en Irlande, l'Espagne fournit deux exemples d'horloges gra-

86. Arnaud Ramière de Fortanier, Documents épigraphiques marseillais du XII' siècle, dans Mélanges André Villard, Marseille, 1975 (= Provence historique, t. 25), p. 295-304, aux p. 299-301 et fig.; C.I.F.M., 14, p. 117-118.

87. On trouve des références épigraphiques au premier jubilé de 1300 en Italie : à Chieti (Abruzzes), à Florence (via Giovanni de Verrazzano), à Rome (San Lorenzo fuori le mura). 


\begin{tabular}{|l|r|r|r|r|r|c|}
\hline & $\begin{array}{c}\text { Avant } \\
1300\end{array}$ & $\begin{array}{c}1300- \\
1349\end{array}$ & $\begin{array}{c}1350- \\
1399\end{array}$ & $\begin{array}{c}1400- \\
1449\end{array}$ & $\begin{array}{c}1450- \\
1499\end{array}$ & TOTAL \\
\hline Calvados & 6 & 16 & 1 & 3 & 1 & 27 \\
\hline Côte d'Or & 22 & 61 & 10 & 4 & 6 & 103 \\
\hline Doubs & 4 & 22 & 9 & 2 & 2 & 39 \\
\hline Essonne & 7 & 14 & 4 & 1 & - & 26 \\
\hline Haute-Saône & 2 & 13 & 2 & 3 & 3 & 23 \\
\hline Marne & 23 & 55 & 8 & 5 & 3 & 94 \\
\hline Oise & 4 & 14 & 2 & 1 & 1 & 22 \\
\hline Pas-de-Calais & 3 & 9 & 2 & 7 & 1 & 11 \\
\hline Seine & 10 & 20 & 7 & 7 & 1 & 45 \\
\hline Seine-et-Marne & 8 & 27 & 6 & 2 & 2 & 45 \\
\hline Seine-Maritime & 12 & 14 & - & 1 & 2 & 29 \\
\hline Yonne & 14 & 20 & 10 & 1 & 2 & 47 \\
\hline TotaL & 115 & 285 & 61 & 37 & 24 & 522 \\
\hline & & & & & & \\
\hline
\end{tabular}

Tableau $n^{\circ}$ 2. - Emploi du calendrier liturgique dans les inscriptions de douze départements.

vées : fragment de marbre trouvé à Santiponte et conservé à Séville, du VII ${ }^{e}$ ou VIII ${ }^{e}$ siècle ([ho]rologium) ${ }^{88}$, et surtout horologium de San Pedro de la Nave, du VIIe siècle, qui donne, deux mois par deux mois, les heures et les "pieds" d'ombre ${ }^{89}$. La Belgique offre un document épigraphique sur ardoises, trouvé dans l'abbaye cistercienne de Villers-en-Brabant, d'un grand

88. Henri Leclercq, "Cadrans solaires", dans Dictionnaire d'archéologie chrétienne et de liturgie, t. II-2 (Paris, 1910), col. 1541-1546, et « Horloge », ibid., t. VI-2 (Paris, 1925), col. 2770-2771.

89. J. Vivès, Inscripciones cristianas..., no 336, p. 115. 
intérêt, car il comporte, à l'usage des sacristains, les instructions pour le fonctionnement de la clepsydre qui réglait la journée monastique ${ }^{90}$.

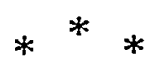

En raison de la masse des inscriptions utilisées, il faudra encore revoir, affiner ces données relatives à la datation dans les inscriptions médiévales de la France, mais l'évolution générale apparaît clairement. On a d'abord vécu sur les systèmes hérités de l'Antiquité. Dates consulaires, puis années de règne, ne permettaient une datation que pour les personnes bien informées et devaient assez vite devenir obscures, sauf pour un cercle restreint. Les années de l'Incarnation, qui avaient de surcroît l'intérêt de répondre à une volonté de christianiser la société, offraient un système simple, qui ne pouvait que l'emporter définitivement. Mais il fallait aussi que s'instaurât une pratique de datation exacte, celle qui ne s'impose vraiment qu'à partir du renouveau de l'écrit, au XII et surtout au XIII ${ }^{e}$ siècle.

L'évolution apparaît comparable en Italie, où l'on trouve, comme en France, l'emploi des années de l'Incarnation dès le IX ${ }^{\mathbf{e}}$ siècle, à Rimini en 818 , à Pola en 857, à Milan en 882, à Parme en 895, à Brescia en 897, à Vérone en 928, à Milan en 900, 956, 963, à Rome en 963, 977, 983. En Allemagne, les exemples relevés en 754-755 à Saint-Géréon de Cologne, en 805 à Saint-Albert de Mayence et à Heppenheim, devraient être examinés de très près, car le mode de datation n'apparaît ensuite qu'à partir du milieu du XI ${ }^{e}$ siècle (1048 à Saint-Étienne de Worms), époque qui pourrait plutôt être son véritable point de départ. On notera qu'en Allemagne le millésime est souvent indiqué en chiffres arabes à partir de 1470 , ce qui ne se rencontre pas en France avant le XVI ${ }^{e}$ siècle. En Espagne, référence est faite à l'année de l'Incarnation à la cathédrale de Pampelune au cours du premier quart du $\mathrm{XII}^{e}$ siècle et quatre inscriptions de la cathédrale de Roda sont datées de cette manière en 1194, et 1197, mais l'ère d'Espagne va régner encore en maître longtemps après cette date dans certains des royaumes de la péninsule ibérique. L'exception est ici le comté de Barcelone, au temps où il a fait partie du royaume franc, avec des exemples d'année

90. Albert D'Haenens, La clepsydre de Villers (1267) : comment on mesurait et vivait le temps dans une abbaye cistercienne au XIII ${ }^{\varepsilon}$ siècle, dans Klösterliche Sachkultur des Spätmittelalters, internazionale Kongress, Krems an der Donau, 18-21 September 1978, Vienne, 1980 (Österreichische Akademie der Wissenschaften, philos. hist. Klasse, Sitzungsberichte, 367 ; Veröffentlichungen des Instituts für mittelalterliche Realienkunde Österreiches, 3), p. 321-352. 
de l'Incarnation, employée seule ou plus généralement associée à l'ère d'Espagne, en $890,914,917,939,942$, etc. ${ }^{91}$.

Pour préciser le jour du mois, le système du calendrier romain a, malgré sa complexité, été employé longtemps, plus longtemps semble-t-il que dans les chartes, sous l'influence probablement des calendriers liturgiques et des obituaires. Il n'est plus guère employé après le premier tiers du XIV ${ }^{\mathrm{e}}$ siècle, avec sans doute une certaine reprise au temps de la Renaissance, qui met en valeur l'héritage de l'Antiquité. Mais dès lors cet usage est artificiel et il reste une exception. L'emploi du quantième du mois à la façon moderne, qui a l'avantage de la simplicité, ne pouvait que s'imposer. Si on le trouve en France au $\mathrm{XI}^{e}$ siècle, sa généralisation ne s'observe qu'à partir de la seconde moitié du XII" siècle. Encore doit-on ici distinguer France du nord et France du midi, cette dernière ne suivant vraiment ce système qu'à partir du XIV ${ }^{e}$ siècle. Cette seconde moitié du XIII ${ }^{e}$ siècle est aussi le moment où ce mode de datation l'emporte à Rome, même si quelques exemples s'y relèvent à compter de 980 . En Espagne, le calendrier romain est constamment employé jusqu'au XIV $\mathrm{XI}^{\mathrm{e}}$ siècle. C'est ainsi le cas des cent quatre-vingtneuf inscriptions du cloître de la cathédrale de Roda entre 1194 et 1413, le cas de toutes les inscriptions des Asturies, tandis que seules deux inscriptions de San Juan de la Peña avant le XVe siècle, en 1301 et 1394, remplacent le calendrier romain par le quantième moderne. En Allemagne, le point de départ du quantième moderne apparaît plus tardif, et le calendrier romain y sera employé beaucoup plus longtemps et assidûment qu'en France ou en Italie.

La datation par une fête religieuse est employée de façon parallèle au quantième moderne, puisque son point de départ ne se situe, sauf rares exceptions, qu'à partir de la seconde moitié du XII ${ }^{e}$ siècle et que son emploi ne se généralise qu'à partir du troisième tiers du XIII ${ }^{e}$ siècle. Là aussi, il se manifeste une différence sensible entre France du nord et France du sud, ce système étant moins répandu et plus tôt délaissé dans la moitié sud du pays. Cette datation doit suivre une évolution assez comparable en Espagne où on commence à la rencontrer vers les mêmes dates qu'en France, à San Miguel de Escalada en 1088, à León en 1122, à Villamartin de Sotoscueva en 1175, à Aguilar de Codès en 1182, à Silos en 1205, à Puylampa en 1224, etc. Ce système a rencontré une particulière faveur en Allemagne, jusqu'aux temps modernes, alors qu'il ne connaît pas une grande vogue en Italie ${ }^{92}$, encore qu'on puisse citer une série d'exemples à Rome à partir de

91. Catalunya romànica, XX, El Barcelonès, el Bax Llobregat, el Maresme, Barcelone, 1992 , p. 247-248, 227, 248, 247, 274, etc.

92. I. Kajanto, Dating in the Latin inscriptions..., p. 58-59. 
$1110^{93}$, beaucoup plus, il est vrai, en référence à un dimanche ou à une station liturgique qu'à une fête de saint.

On ne peut douter qu'une étude portant sur l'ensemble de la chrétienté montrerait des différences sensibles dans la chronologie et la géographie des différents systèmes de datation dans les inscriptions médiévales. Il faudrait encore mesurer le poids du conservatisme et de l'influence de la liturgie dans les épitaphes ou les inscriptions de dédicace, des rapports avec la pratique des chartes, les changements qu'a pu apporter le remplacement du latin par la langue vulgaire. On ne pouvait tout aborder dans un premier essai global. L'étude de la chronologie s'impose pour l'épigraphiste, qui peut y trouver des éléments de critique sur la datation des textes qu'il étudie. Elle est aussi le reflet de sensibilités, de cultures, de spiritualités qui varient selon les temps et les lieux, et par là elle intéresse aussi directement l'histoire.

Robert Favreau.

93. À S. Matteo in Merulana, S. Maria in Aquiro en 1179, S. Nicolas de Funari en 1180 , S. Giovanni a Porta latina en 1190, S. Eustachio en 1196, etc. 